\title{
DESIGN DEVELOPMENT OF DYNAMOMETER WATER ABSORBER
}

\author{
Karlis Banis \\ Latvia University of Life Sciences and Technologies, Latvia \\ k.banis@yahoo.com
}

\begin{abstract}
The paper investigates the effect of several design parameters of a dynamometer water absorber (brake). This type of absorber is widely used in dynamometer testing of various applications due to its relatively inexpensive and power-dense design. Such applications include the bench testing of motorcycle, automotive, heavy equipment and even marine engines. The drawback of water absorbers is their complicated control characteristics making them undesirable for use in precision tests. The aim of this study is to evaluate the change in efficiency and linearity of torque absorption with respect to design parameters. Steady state computational fluid dynamics analysis at constant inlet flow rate was carried out in SolidWorks Flow Simulation environment. Twenty-one different design parameters were tested at three different operation speeds and compared to the baseline test. The output absorption torque values were graphed and analyzed, coefficient of linearity and low speed absorption torque was compared. With the primary focus on low speed torque absorption efficiency, it was found that certain values of the tested parameters, such as rotor and stator blade angles, fillet and rotor-to-stator gap, have a substantial effect on both the linearity and efficiency of the absorber, but reversed values of the same parameters were found to degrade the efficiency in most cases. Without considering undesirable design parameters, the highest low speed torque absorption efficiency gain was achieved by filleting the rotor blades or slanting the stator blades $-15^{\circ}$ around the axis of rotation.
\end{abstract}

Keywords: computational fluid dynamics, water absorber, dynamometer.

\section{Introduction}

As the age of global environment protection is imposing ever-increasing regulations on emissions and fuel efficiency of internal combustion engines, it is always equally important that appropriate testing can be ensured in order to aid the development process or validate the end-result. In the case of internal combustion engines, either gasoline, diesel, or natural gas, test benches or dynamometers are often used for this purpose. They are classified as chassis or engine benches that can further be described as either inertia or load type. The most precise variants are load dynamometers that use a type of absorber - either mechanical, electrical or hydraulic. While electrical absorbers are known for their accurate control characteristics, they are often very expensive and unaffordable for smaller companies. Water absorber is a good alternative that provides high power density, allowing the system to be relatively small, increasing portability and decreasing space requirements [1]. Furthermore, because the power application is by means of pressurized fluid, there are no high current electrical circuits anywhere near the engine. This significantly lowers any electromagnetic interference with low voltage and current sensors that may be on the engine, such as pressure transducers or accelerometers [2]. The rotor is connected to the test engine creating a pumping action of water and directing the flow over stator vanes in a toroidal vortex pattern and creating a torque reaction through the absorbers casing [3]. The rate of change of angular momentum of the water is directly related to the absorption torque of the rotor [4]. The absorption torque is also influenced by friction along the surfaces of walls and vanes as well as the turbulent flow [5]. The absorbed torque is converted to heat and taken away by the drain water. In general, the torque capacity of the water absorber can be considered as a fifth power function of the rotor diameter and the power capacity as a third power function of the rotor diameter [6], but a more accurate calculation of brake performance can only be obtained by 3D viscous internal flow field analysis [7]. The disadvantages of water absorbers include non-linear (mostly superlinear) behavior, as well as oscillations in the measured torque due to oscillations in the water flow requiring a sophisticated rotor design [8;9]. Because of these disadvantages water absorbers are not recommended for dynamic testing and road simulation purposes [10], although some examples of advanced water absorber control systems exist and operate on a sufficient level for lower budget test facilities.

\section{Materials and methods}

The object of investigation is a blade type water absorber with a two-sided rotor of diameter 200 $\mathrm{mm}$. The basic design is symmetric, meaning that blades are positioned on both sides of the rotor, with each of the sides facing to an individual stator. The two stator halves each comprise a water inlet at the 
upper portion and a water outlet at the bottom. General design parameters are chosen according to similar water absorbers available on the market and illustrated in Figure 1.
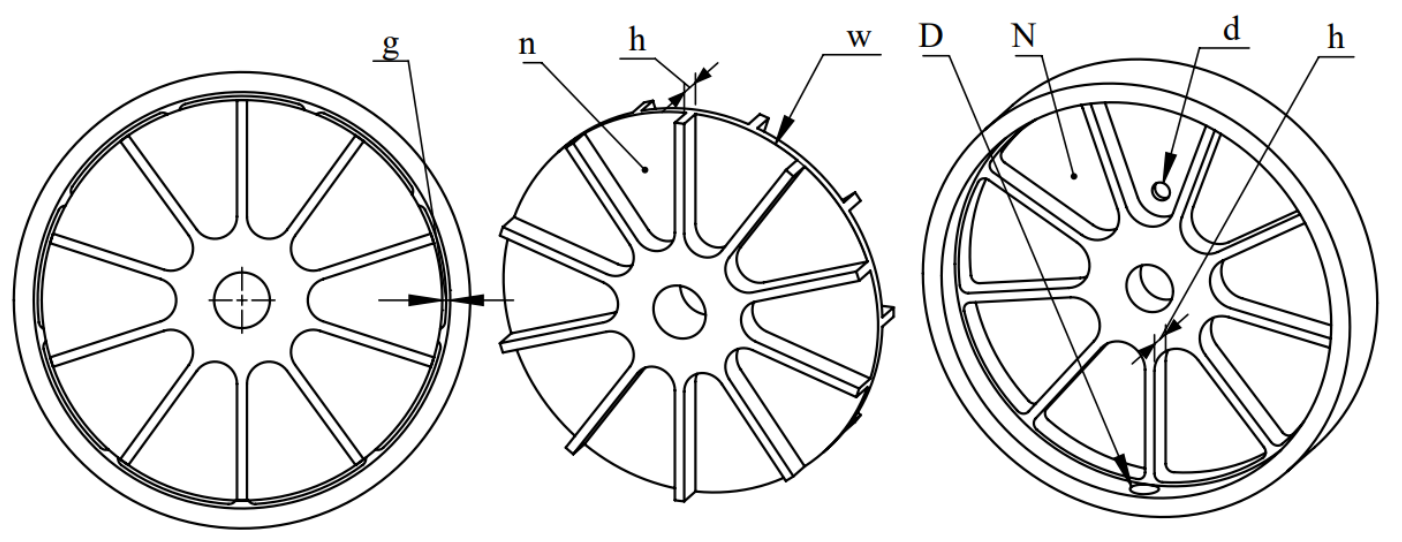

Fig. 1. Basic rotor and stator design: $\mathrm{g}$ - rotor-to-stator gap $(4 \mathrm{~mm}) ; \mathrm{n}$ - number of rotor pockets (10); $\mathrm{h}$ - rotor and stator blade width $(16 \mathrm{~mm}) ; \mathrm{w}$ - rotor diameter $(200 \mathrm{~mm}) ; \mathrm{D}$ - water outlet diameter (14.5 mm); $\mathrm{N}$ - number of stator pockets (9); $\mathrm{d}$ - water inlet diameter $(9.5 \mathrm{~mm})$

By its principle of operation, the water absorber can be considered as a very inefficient water pump, where majority of the mechanical input is converted to heat, but only a small portion of it is used to move the water. It is known that the absorption torque of the rotor is superlinear with respect to its speed of rotation. This makes the water absorber undesirable for use in low speed conditions where some sort of transmission mechanism would be necessary. In order to test the low speed efficiency and the linearity of torque absorption, a computation fluid dynamics study is set up, using the design parameters, as seen in Figure 2. The values of said parameters are chosen based on the limits of the general design and listed in Table 1.
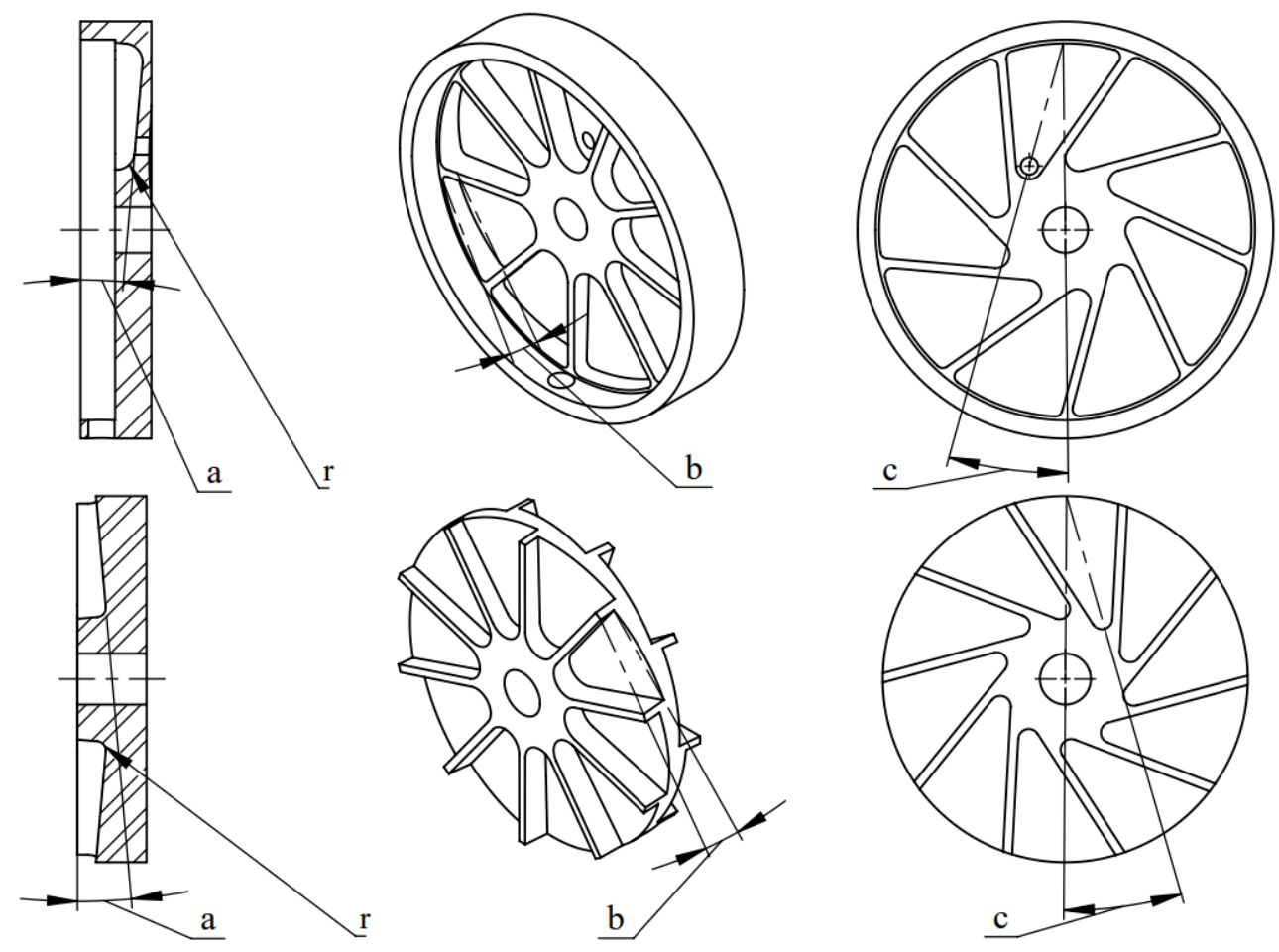

Fig. 2. Rotor and stator design parameters: $r$ - blade fillet; $a$ - angle $1 ; \mathrm{b}$ - angle 2; $\mathrm{c}$ - angle 3

The flow simulation is set up in SolidWorks Flow Simulation 2018 environment using steady state analysis with a rotating frame - the rotor. Water is chosen as the simulation fluid and supplied to the two water inlet boundaries (see Figure $1-\mathrm{d}$ ) at constant flow rate $-201 \cdot \mathrm{min}^{-1}$ and a constant temperature of $30{ }^{\circ} \mathrm{C}$. The water outlet boundaries (see Fig. $1-\mathrm{D}$ ) are defined as constant pressure openings of temperature $30^{\circ} \mathrm{C}$ and the environment pressure $-101.325 \mathrm{kPa}$. Three different speeds of 
rotation are assigned to the rotor $-2,000 \mathrm{~min}^{-1}$ (low speed), 5,000 $\mathrm{min}^{-1}$ (medium speed) and $8,000 \mathrm{~min}^{-1}$ (high speed). The resulting frictional torque around the axis of rotation is measured on all the faces of the rotor. The resulting 3D mesh is shown in Figure 3.

Table 1

Design parameters

\begin{tabular}{|c|c|c|c|c|c|c|c|}
\hline \multirow[t]{2}{*}{ Design } & \multirow[t]{2}{*}{ Object } & $\begin{array}{l}\text { Blade } \\
\text { width }\end{array}$ & $\begin{array}{l}\text { Rotor-to- } \\
\text { stator gap }\end{array}$ & $\begin{array}{l}\text { Blade } \\
\text { fillet }\end{array}$ & Angle 1 & Angle 2 & Angle 3 \\
\hline & & \multicolumn{3}{|c|}{$\mathrm{mm}$} & \multicolumn{3}{|c|}{ degrees } \\
\hline $\begin{array}{c}\mathrm{A} \\
\text { (baseline) }\end{array}$ & $\begin{array}{c}\text { Rotor \& } \\
\text { Stator }\end{array}$ & 16 & 4 & 0 & 0 & 0 & 0 \\
\hline B & \multirow{2}{*}{$\begin{array}{l}\text { Rotor \& } \\
\text { Stator }\end{array}$} & 12 & \multirow{2}{*}{4} & \multirow{2}{*}{0} & \multirow{2}{*}{0} & \multirow{2}{*}{0} & \multirow{2}{*}{0} \\
\hline $\mathrm{C}$ & & 20 & & & & & \\
\hline $\mathrm{D}$ & \multirow{2}{*}{$\begin{array}{c}\text { Rotor \& } \\
\text { Stator }\end{array}$} & \multirow{2}{*}{16} & 2 & \multirow{2}{*}{0} & \multirow{2}{*}{0} & \multirow{2}{*}{0} & \multirow{2}{*}{0} \\
\hline E & & & 8 & & & & \\
\hline $\mathrm{F}$ & \multirow{2}{*}{ Rotor } & \multirow{2}{*}{16} & \multirow{2}{*}{4} & 5 & \multirow{2}{*}{0} & \multirow{2}{*}{0} & \multirow{2}{*}{0} \\
\hline $\mathrm{G}$ & & & & 10 & & & \\
\hline $\mathrm{H}$ & \multirow{2}{*}{ Rotor } & \multirow{2}{*}{16} & \multirow{2}{*}{4} & \multirow{2}{*}{0} & 5 & \multirow{2}{*}{0} & \multirow{2}{*}{0} \\
\hline $\mathrm{I}$ & & & & & -5 & & \\
\hline $\mathrm{J}$ & \multirow{2}{*}{ Rotor } & \multirow{2}{*}{16} & \multirow{2}{*}{4} & \multirow{2}{*}{0} & \multirow{2}{*}{0} & 5 & \multirow{2}{*}{0} \\
\hline $\mathrm{K}$ & & & & & & -5 & \\
\hline $\mathrm{L}$ & Rotor & 16 & 4 & 0 & 0 & 0 & 15 \\
\hline M & NOUTO & 10 & 4 & 0 & 0 & 0 & -15 \\
\hline $\mathrm{N}$ & Stator & 16 & 4 & 5 & 0 & 0 & 0 \\
\hline $\mathrm{O}$ & Stator & 10 & 4 & 10 & 0 & 0 & 0 \\
\hline $\mathrm{P}$ & Stator & 16 & 4 & 0 & 5 & 0 & 0 \\
\hline$Q$ & Stator & 10 & 4 & 0 & -5 & 0 & 0 \\
\hline $\mathrm{R}$ & Stator & 16 & 4 & 0 & 0 & 5 & 0 \\
\hline $\mathrm{S}$ & Niator & 10 & 4 & $\sigma$ & 0 & -5 & 0 \\
\hline $\mathrm{T}$ & Stator & 16 & 4 & 0 & 0 & () & 15 \\
\hline $\mathrm{U}$ & Statol & 10 & 4 & 0 & 0 & 0 & -15 \\
\hline
\end{tabular}

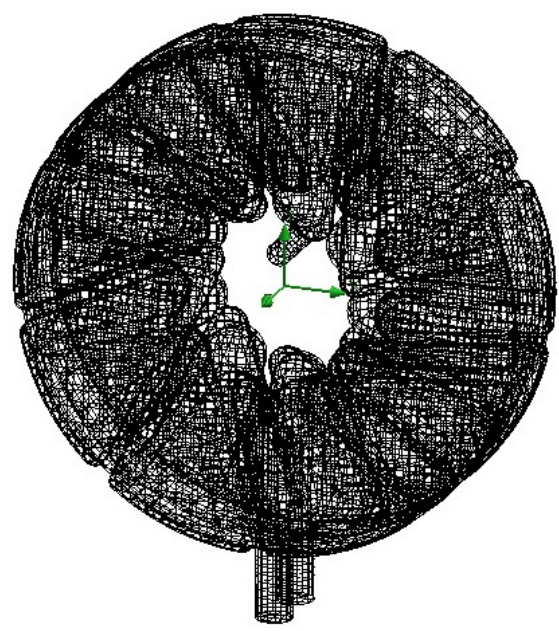

Fig. 3. 3D Simulation mesh

\section{Results and discussion}

The flow simulation results (see Table 2) show the absorption torque dependency on the rotation speed of superlinear nature - the relative absorption torque increase is of a larger magnitude than the relative increase in the rotation speed. This can be seen in Figure 4 showing designs exceling in this aspect the most. This could possibly be explained by the kinetic energy equation of moving objects, in 
this case - water. Equation 1 shows the relation between the kinetic energy of moving objects, their mass and velocity. It can be seen, that the velocity has a much more substantial effect on the kinetic energy, as it is expressed as a squared value, meaning that the kinetic energy is increasing faster than the velocity or speed of rotation.

$$
K=\frac{m \cdot v^{2}}{2},
$$

$$
\begin{array}{ll}
\text { where } & K-\text { kinetic energy, } \mathrm{J} \\
& m-\text { mass, } \mathrm{kg} \\
& v-\text { velocity, } \mathrm{m} \cdot \mathrm{s}^{-1}
\end{array}
$$

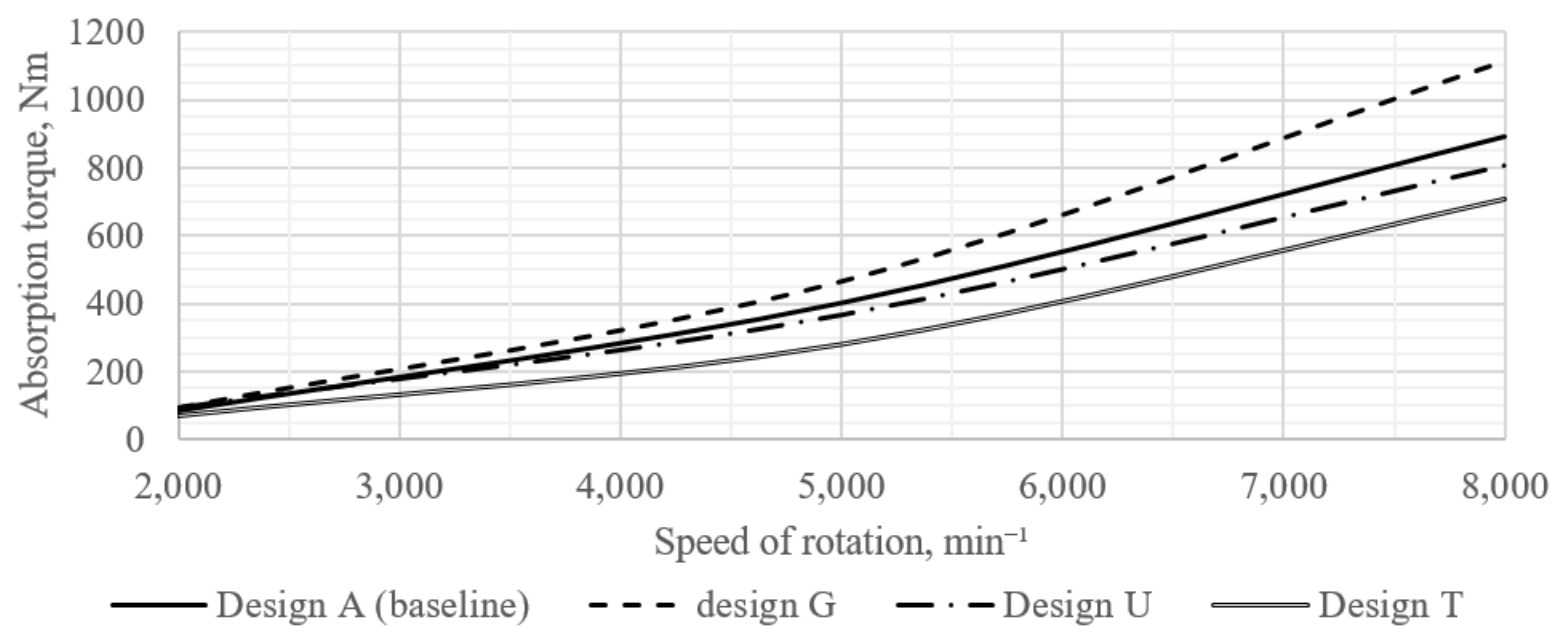

Fig. 4. Absorption torque results

The most noticeable effect is observed by the change in the rotor-to-stator gap (see Fig. $1-\mathrm{g}$ ): $56.78 \%$ low speed torque absorption gain, when decreasing it from $4 \mathrm{~mm}$ to $2 \mathrm{~mm}$ (Design D), and $19.41 \%$ loss, when increasing it from $4 \mathrm{~mm}$ to $8 \mathrm{~mm}$ (Design E). While better results can be obtained with a smaller rotor-to-stator gap, in practice however, an overly small gap is not recommended due to the risk of choking the water drainage, which limits the volume flow rate of water and heat removal, while delaying the absorbers response to changes in water supply. A rather unexpected outcome is the effect of the blade width on low speed absorption torque and the coefficient of linearity $\left(\mathrm{R}^{2}\right)$. The highest absorption torque and $\mathrm{R}^{2}$ value were obtained on the baseline test and suffered a decrease in efficiency and linearity when either decreasing or increasing the blade width (Designs B and C).

Figure 5 shows the relative low speed torque absorption between the tested designs in percent over the baseline test. The presence of the blade fillet was observed to be the most efficient when used on the rotor (Designs F and G) instead of the stator (Designs N and O). An increase in the fillet radius (see Fig. $2-r$ ) tends to increase the absorbers low speed efficiency with a slight compromise of linearity. Different blade angles (see Fig. $2-$ a) were observed to be the most beneficial when used on the stator (Designs $\mathrm{P}-\mathrm{U}$ ), while leading to either negligible improvements or extensive low speed efficiency loss when applied to the rotor (Designs $\mathrm{H}-\mathrm{M}$ ), but showing different results in high speed efficiency (see Table 2).

Table 2

Flow simulation results

\begin{tabular}{|c|c|c|c|c|}
\hline \multirow{2}{*}{ Design } & \multicolumn{4}{|c|}{ Absorption torque, Nm } \\
\cline { 2 - 5 } & $\mathbf{2 , 0 0 0} \mathbf{~ m i n}^{-\mathbf{1}}$ & $\mathbf{5 , 0 0 0} \mathbf{~ m i n}^{-1}$ & $\mathbf{8 , 0 0 0} \mathbf{~ m i n}^{-1}$ & $\boldsymbol{R}^{\mathbf{2}}$ \\
\hline A (Baseline) & 81.9 & 401.3 & 893.4 & 0.985 \\
\hline B & 77.3 & 277.2 & 756.9 & 0.947 \\
\hline C & 72.0 & 294.5 & 696.3 & 0.973 \\
\hline D & 128.4 & 473.1 & $1,229.5$ & 0.956 \\
\hline E & 66.0 & 308.3 & 762.0 & 0.970 \\
\hline F & 85.8 & 416.2 & 928.9 & 0.985 \\
\hline
\end{tabular}


Table 2 (continued)

\begin{tabular}{|c|c|c|c|c|}
\hline \multirow{2}{*}{ Design } & \multicolumn{4}{|c|}{ Absorption torque, $\mathrm{Nm}$} \\
\hline & $2,000 \mathrm{~min}^{-1}$ & $5,000 \mathrm{~min}^{-1}$ & 8,000 min $^{-1}$ & $R^{2}$ \\
\hline $\mathrm{G}$ & 92.5 & 464.8 & $1,114.0$ & 0.976 \\
\hline $\mathrm{H}$ & 82.5 & 321.5 & 804.8 & 0.963 \\
\hline $\mathrm{I}$ & 79.9 & 399.9 & $1,035.1$ & 0.965 \\
\hline $\mathrm{J}$ & 83.2 & 364.7 & 865.7 & 0.974 \\
\hline $\mathrm{K}$ & 77.0 & 388.4 & 926.0 & 0.977 \\
\hline $\mathrm{L}$ & 71.6 & 372.4 & 930.0 & 0.971 \\
\hline $\mathrm{M}$ & 75.0 & 399.9 & 995.1 & 0.972 \\
\hline $\mathrm{N}$ & 81.5 & 419.9 & 994.5 & 0.978 \\
\hline $\mathrm{O}$ & 85.5 & 379.4 & $1,052.1$ & 0.951 \\
\hline $\mathrm{P}$ & 83.2 & 365.0 & $1,039.3$ & 0.947 \\
\hline$Q$ & 87.5 & 379.9 & 862.0 & 0.980 \\
\hline $\mathrm{R}$ & 77.6 & 374.7 & 971.8 & 0.964 \\
\hline $\mathrm{S}$ & 77.9 & 403.3 & 896.9 & 0.986 \\
\hline $\mathrm{T}$ & 70.5 & 280.8 & 707.6 & 0.963 \\
\hline $\mathrm{U}$ & 90.8 & 364.5 & 805.3 & 0.982 \\
\hline
\end{tabular}

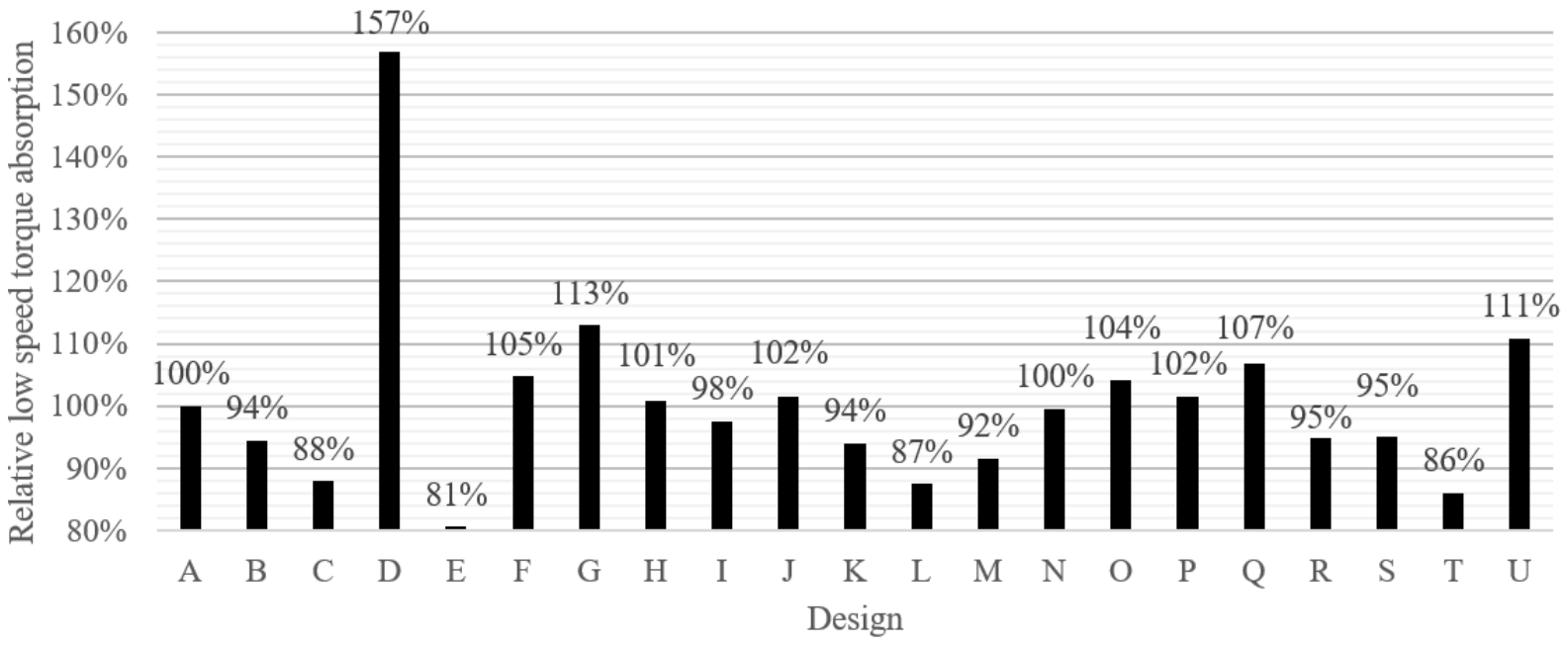

Fig. 5. Low speed torque absorption comparison against baseline

\section{Conclusions}

1. Twenty-one water absorber designs were tested and the relative torque absorption was measured ranging in magnitude from $80.6 \%$ up to $156.8 \%$ against the baseline absorption test at low speeds $\left(2,000 \mathrm{~min}^{-1}\right)$.

2. Decreasing the rotor-to-stator gap from $4.0 \mathrm{~mm}$ to $2.0 \mathrm{~mm}$ substantially improves the efficiency of the water absorber $(+56.8 \%$ increase in low speed efficiency and $+37.6 \%$ increase in high speed efficiency), using an overly small gap is not recommended due to the risk of choking the water drain. Such condition can significantly reduce or delay the response of the absorber to changes in the water flow making the operation more unpredictable.

3. Without considering the changes in the rotor-to-stator gap, the most substantial improvements in low speed efficiency were achieved by the design $\mathrm{G}$ using a $10 \mathrm{~mm}$ fillet on the rotor $(+12.9 \%)$ and the design $U$ using stator blades slanted $-15^{\circ}$ around the axis of rotation $(+10.9 \%)$ with the slight compromise of linearity ( $\mathrm{R}^{2}-1.9 \%$ and $-0.3 \%$ respectively). The highest linearity $-\mathrm{R}^{2}$ was achieved by the design $\mathrm{E}$ using $8 \mathrm{~mm}$ rotor-to-stator gap (+0.2\%) and the design $\mathrm{S}$ using stator blades slanted $-5^{\circ}$ around the radial axis $(+0.3 \%)$.

4. During the flow simulation, the solver had exhibited signs of low stability requiring a high number of iterations until an acceptable result was reached. To increase the accuracy of this study, 
the calculations should be performed on a very powerful computer, the number of iterations greatly increased, and the inlet boundary conditions set to "static pressure" of a value that is produced at the outlet of the pump used.

\section{Acknowledgements}

This publication has been prepared within the framework of the Latvia University of Life Sciences and Technologies project "Z25 - Innovative improvements of gas exchange system efficiency for internal combustion engines" of the programme "Strengthening of scientific capacity $2018 "$

\section{References}

[1] Holland M. A., Harmeyer K., Lumkes J. H. Design of a high-bandwidth, low-cost hydrostatic absorption dynamometer with electronic load control. SAE Technical Paper No. 2009-01-2846, 2009.

[2] Babbitt G. R., Moskwa J. J. Hardware Implementation Details and Test Results for a HighBandwith, Hydrostatic Transient Engine Dynamometer System. SAE Technical Paper No. 970025, 1997.

[3] Killedar J. S. Dynamometer: theory and application to engine testing. Xlibris Corporation, 2012. $607 \mathrm{p}$.

[4] Raine J. K., Hodgson P. G. Computer Simulation of a Variable Fill Hydraulic Dynamometer. Part 1: Torque Absorption Theory and the Influence of Working Compartment Geometry on Performance. Proceedings of the Institution of Mechanical Engineers, Part C: Mechanical Engineering Science, May 1, 1991, pp. 155-163.

[5] Vetr M., Passenbrunner T. E., Trogmann H., Ortner P., Kokal H., Schmidt M., Paulweber M. Control oriented modeling of a water brake dynamometer. Proceedings of IEEE International conference "Control Applications", September 8-10, 2010, Yokohama, Japan, pp. 222-227.

[6] Bettes H., Hancock B. Dyno testing and tuning. CarTech Inc., 2008. 144 p.

[7] Wei W., Yan Q. D., Wu J. Y. Research on numerical investigation and test verification of brake performance in a hydrodynamic tractor-retarder assembly. Advanced Materials Research, vol. 97, 2010, pp. 3357-3361.

[8] Passenbrunner T. E., Sassano M., Trogmann H., del Re L., PaulweberM., SchmidtM., KokalH. Inverse torque control of hydrodynamic dynamometers for combustion engine test benches. Proceedings of the American Control Conference, June 29 - July 1, 2011, San Francisco, CA, USA, pp. 4598-4603.

[9] Van Den Braembussche R. A., Malys H. Dynamic stability of a water brake dynamometer. Journal of engineering for gas turbines and power, vol. 120(1), 1998, pp. 89-96.

[10] Schürmann D., Johnsson L., Berg W. Calibration of Chassis Dynamometers for Emission-and Fuel Economy Testing Using Wheel Torque Meters. SAE Technical Paper No. 800400, 1980. 